\title{
Distribution of freshwater red algal family Lemaneaceae (Rhodophyta) in the Czech Republic: an update
}

\author{
Pavel KuČERA, Vít Grulich, Markéta FránKová \& Petr Bureš
}

Institute of Botany and Zoology, Masaryk University Brno, Kotlářská 2, 61137 Brno, Czech Republic, e-mail: kucerap@email.cz

\begin{abstract}
Macroscopic red algae of the family Lemaneaceae are reported from several localities in the Czech Republic. Published data are sparse due to difficulty in species identification and little interest in this group of algae in the past. The Lemaneaceae were sampled throughout the Czech Republic. The new localities are reported as follows: 18 for Lemanea fluviatilis and 5 for Paralemanea catenata. Results from this study suggest that $L$. fluviatilis is widespread and inhabits streams from a wide altitudinal gradient, whereas $P$. catenata has a more restricted distribution. Lemanea tortulosa appears to be very rare having been identified from a single site.
\end{abstract}

Key words: Czech Republic, distribution, Lemanea, Lemaneaceae, Paralemanea, red algae

\section{Introduction}

The freshwater red algal family Lemaneaceae is a characteristic group in running waters with the world-wide distribution. Although some species are common, others seem to be quite rare and limited to a special biotop or water type (ELORANTA \& KWANDRANS 2002). Recently, much of the research on this group has been concentrated in the North America (e.g. Vis \& Sheath 1992, Carmona \& NeCCHI 2002) and in Brazil (NeCCHI \& ZuCCHI 1995). Some studies have been done in Europe: in Sweden by Israelson (1942) and in Poland by STARMACH (1977), in Finnland by Eloranta \& Kwandrans (2002), in the Czech Republic by LedERER \& LHOtský (2001) and KuČERA \& MARVAn (2004). However, more detailed studies of taxonomy and distribution for the freshwater Lemaneaceae in Europe are still needed.

The freshwater red algae of the family Lemaneaceae are characterized by an uniaxial cartilagineous and pseudoparenchymatous gametophyte thallus with in ternal carposporophytes (VIS \& ShEATH 1992). These authors raised the traditionally distinguished subgenera (Lemanea and Paralemanea) of the genus Lemanea to genera. These genera can be separated on the basis of the following characters (VIS \& SHEATH 1992, SheAth et al. 1996): Paralemanea includes plants that lack stalks and hair cells in the inner cortex. Axial filaments are surrounded by cortical filaments. Simple ray cells consist of two layers, the proximal one does not touch the outer cortex and the distal one is generally "Y" branched and connected to the cortex. Nodal spermatangial sori are arranged in rings. Lemanea has axial (but not cortical) filaments, hair cells in the inner cortex and T- or L-shaped ray cells, which are closely applied to the outer cortex. Nodal spermatangial sori are arranged in patches.

The first references on the occurence of Lemaneaceae in the Czech Republic were published by HANSGIRG (1886, 1892), PASCHER (1903), Maloch (1937), WÜNsCh (1937) and Cyrus (1947) (PoulíčKovÁ et al. 2004). LhotskÝ \& RosA (1955) described 14 localities for Lemanea fluviatilis and 8 localities for Lemanea torulosa. However, the species descriptions by these authors were not sufficiently detailed to verify these reports using the current taxonomy. A recent systematic investigation of Lemaneaceae noted three species in the Czech Republic: Lemanea fluviatilis, L. torulosa and Paralemanea catenata (KUČERA \& MARVAN 2004). The Lemaneaceae inhabit fast flowing and turbulent water and are rare in the Czech Republic (GARDAVSKÝ et al. 1995), as they are sensitive to levels of pollution. Therefore, the aim of this work is to provide data on the occurence of the freshwater red algal family Lemaneaceae in the Czech Republic, to verify previously published occurence records and report new localities. 
Table 1: Geographical characteristics of localities. The latitude and longitude were obtained using GeoBáze software. New localities (previously not reported) are marked with an asterisk.

\begin{tabular}{|c|c|c|c|c|}
\hline No. & Locality & River & Substrate & Latitude and longitude \\
\hline & \multicolumn{4}{|l|}{ Lemanea fluviatilis } \\
\hline 1 & Valšov & Moravice & weir & $49 ; 55 ; 25+17 ; 27 ; 03$ \\
\hline 2 & Annín & Hučivá Desná & stones & $50 ; 06 ; 52+17 ; 06 ; 30$ \\
\hline 3 & Karlova Studánka & Bílá Opava & stones (waterfall) & $50 ; 04 ; 38+17 ; 17 ; 12$ \\
\hline 4 & Kozlov & Otava & stones & $49 ; 17 ; 54+13 ; 45 ; 04$ \\
\hline 5 & Čepice & Otava & stones & $49 ; 16 ; 02+13 ; 35 ; 55$ \\
\hline 6 & Čeňkova pila & Otava & stones & $49 ; 06 ; 30+13 ; 29 ; 33$ \\
\hline 7 & Žichovice & Otava & stones & $49 ; 16 ; 24+13 ; 37 ; 05$ \\
\hline 8 & Železná ruda & ̌̌ezná & stones & $49 ; 08 ; 07+13 ; 13 ; 27$ \\
\hline 9 & Švařec** & Svratka & stones & $49 ; 31 ; 10+16 ; 20 ; 38$ \\
\hline 10 & Raškov* & Morava & stones & $50 ; 01 ; 51+16 ; 54 ; 07$ \\
\hline 11 & Tuřice / Předměřice nad Jizerou* & Jizera & stones & $50 ; 15 ; 03+14 ; 46 ; 28$ \\
\hline 12 & Heřmaň* & Blanice & stones & $49 ; 14 ; 18+14 ; 08 ; 14$ \\
\hline 13 & Nemětice* & Volyňka & stones & $49 ; 11 ; 50+13 ; 53 ; 08$ \\
\hline 14 & Pěkná* & Vltava & stones & $48 ; 51 ; 07+13 ; 55 ; 13$ \\
\hline 15 & Rešovské vodopády* & Huntava & stones (waterfall) & $49 ; 52 ; 52+17 ; 12 ; 37$ \\
\hline 16 & Vír* & Bystřice & stones & $49 ; 33 ; 04+16 ; 18 ; 32$ \\
\hline 17 & Vír* & Svratka & weir & $49 ; 33 ; 40+16 ; 18 ; 28$ \\
\hline 18 & Dalečín* & Svratka & weir & $49 ; 35 ; 33+16 ; 14 ; 56$ \\
\hline 19 & Malá Morava* & Malá Morava & stones & $50 ; 05 ; 13+16 ; 50 ; 32$ \\
\hline 20 & Velká Úpa* & Javoří potok & stones & $50 ; 40 ; 50+15 ; 45 ; 07$ \\
\hline 21 & Pec pod Sněžkou* & Úpa & stones & $50 ; 42 ; 00+15 ; 44 ; 11$ \\
\hline 22 & Dolní Malá Úpa* & Malá Úpa & stones & $50 ; 40 ; 57+15 ; 48 ; 22$ \\
\hline 23 & Špindlerův Mlýn* & Labe & weir, stones & $50 ; 43 ; 31+15 ; 36 ; 22$ \\
\hline 24 & Vrchlabí ${ }^{*}$ & Labe & stones & $50 ; 38 ; 45+15 ; 36 ; 24$ \\
\hline 25 & Skoronice* & Malše & stones & $48 ; 42 ; 39+14 ; 29 ; 04$ \\
\hline 26 & Černý Kř́íž & Studená Vltava & stones & $48 ; 51 ; 38+13 ; 51 ; 23$ \\
\hline 27 & Stř́ibro & Mže & weir & $49 ; 44 ; 59+12 ; 59 ; 48$ \\
\hline 28 & Radčice & Mže & weir & $49 ; 45 ; 37+13 ; 19 ; 47$ \\
\hline \multirow[t]{2}{*}{29} & Plzeň-Kalikovský mlýn & Mže & weir & $49 ; 45 ; 02+13 ; 21 ; 53$ \\
\hline & Lemanea torulosa & & & \\
\hline \multirow[t]{2}{*}{30} & Žákava & Bradava & weir & $49 ; 38 ; 06+13 ; 31 ; 29$ \\
\hline & Paralemanea catenata & & & \\
\hline 31 & Kunín - Valcha* & Odra & stones & $49 ; 39 ; 48+17 ; 58 ; 59$ \\
\hline 32 & Plasy* & Střela & weir & $49 ; 56 ; 08+13 ; 23 ; 13$ \\
\hline 33 & Žlutice* & Střela & stones & $50 ; 05 ; 11+13 ; 08 ; 48$ \\
\hline 34 & Štoutov* & Velká Trasovka & stones & $50 ; 07 ; 16+13 ; 11 ; 43$ \\
\hline 35 & Náměšt’ nad Oslavou* & Oslava & stones & $49 ; 14 ; 28+16 ; 07 ; 54$ \\
\hline 36 & Čepinec & Bradlava & stones & $49 ; 28 ; 09+13 ; 29 ; 15$ \\
\hline 37 & Mlynářovice & Bradlava & stones & $49 ; 25 ; 43+13 ; 27 ; 48$ \\
\hline 38 & Hvížd’alka & Bradava & stones & $49 ; 36 ; 47+13 ; 37 ; 04$ \\
\hline 27 & Stř́íbro & Mže & weir & $49 ; 44 ; 59+12 ; 59 ; 48$ \\
\hline 28 & Radčice & Mže & weir & $49 ; 45 ; 37+13 ; 19 ; 47$ \\
\hline 29 & Plzeň-Kalikovský mlýn & Mže & weir & $49 ; 45 ; 02+13 ; 21 ; 53$ \\
\hline 39 & Vranov nad Dyjí & Dyje & weir & $48 ; 53 ; 37+15 ; 48 ; 48$ \\
\hline 40 & Devět Mlýnů & Dyje & weir & $48 ; 49 ; 01+15 ; 58 ; 55$ \\
\hline 41 & Znojmo & Dyje & stones & $48 ; 51 ; 12+16 ; 02 ; 36$ \\
\hline
\end{tabular}




\section{Material and methods}

Stream sites were visited during 2002-2006 in different regions of the Czech Republic. All samples were collected from running water with turbulent flow (weirs and riffles). The samples were preserved in $4 \%$ formaldehyde and studied using a microscope equipped with equipped with Lucia Image Analysis. For the identification of species, Vis \& SHEATH (1992) and KuČERA \& MARVAN (2004) were used.

\section{Results and discussion}

The Lemaneaceae were found on stony substrates in running turbulent waters, on weirs or in waterfalls (Table 1, Fig. 1).

Lemanea fluviatilis (Linnaeus) C. Agardh 1824 (bas. Conferva fluviatilis LinNAEus 1753, syn. Sacheria fluviatilis (LinNAEus) SiRodot 1872) was the most frequently encountered species in the Czech Republic. Lederer \& SoukupovÁ (2002) described this species from mountain streams and rivers. According to our observations, this species occurs not only in mountains. In this investigation, it was found at altitudes ranging from 305 to 888 $\mathrm{m}$. The species was observed on stony substrates (boulders and cobbles) in riffles, on weirs or in waterfalls (Table 1), which were partly shaded or well illuminated. At certain localities, it was growing with $P$. catenata (e.g. locality Strúbro). According to Vis \& Sheath (1992), this species has the widest range of distribution of Lemaneaceae in North America, which could be due to a greater tolerance of conditions. ElORANTA \& KWANDRANS (1996) described this species from the wide range of flow velocity $\left(20-190 \mathrm{~cm} \cdot \mathrm{s}^{-1}\right)$ without any clear preference. JOHANSSON (1982) recorded this species from small lowland rivers with rather high conductivity, high colour, but small insolation. FILKIN \& VIS (2004) recorded the highest percent cover in biotops with high flow velocity and low water depth.

Lemanea torulosa (Roth) C. AgARDH 1814 (bas. Conferva torulosa Rотн 1797) was found only at a single locality in the Czech Republic - Žákava, a stony weir shaded by trees in river Bradava (KuČERA \& MARVAN 2004). In the past, this species was collected in the Sumava Mountains (GARDAVSKÝ et al. 1995).

Paralemanea catenata (KüTZING) VIS et SHEATH 1992 (bas. Lemanea catenata KÜTZING 1843, syn. Lemanea annulata KützING 1843, Lemanea nodosa KützING 1843, Lemanea pleocarpa AtKInson 1931) was found on stony substrates or on weirs (Table 1). The plants grow on stony weirs together with mosses and Cladophora glomerata. The species occured in shaded (localities Čepinec, Mlynářovice, Hvížd'alka) or well illuminated areas (Vranov nad Dyjí, Devět mlýnů, Znojmo). CARMONA \& NeCCHI (2002) recorded this species from shallow (depth $1-60 \mathrm{~cm})$, cold $\left(12-16^{\circ} \mathrm{C}\right)$, and moderate to fast

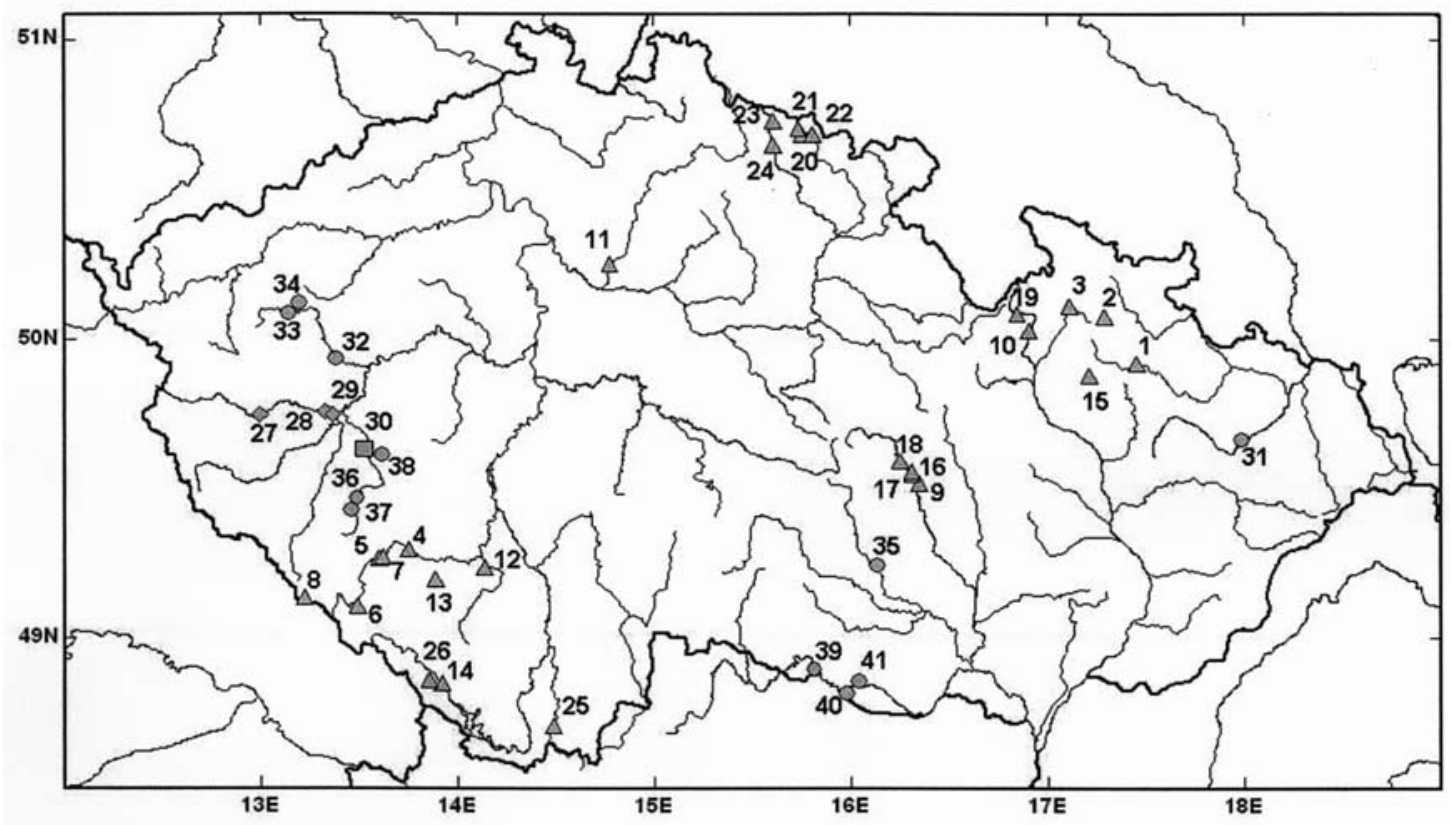

Fig. 1. Map showing the localities of Lemaneaceae in the Czech Republic. Numbers correspond to those in Table 1. $\mathbf{\Delta}-L e$ manea fluviatilis, $\bullet-$ Paralemanea catenata $\bullet-$ L. fluviatilis and P. catenata, - - Lemanea torulosa. 
flowing waters $\left(>35 \mathrm{~cm} \cdot \mathrm{s}^{-1}\right)$. All localities in our study have the same characteristics as reported in the literature (e.g. Eloranta \& Kwandrans 2002, CARMONa \& NeCCHI 2002).

\section{Acknowledgements}

Authors acknowledge especially Petr Marvan and Rodan Geryš for help. The study has been supported by a research project MSM0021622416.

\section{References}

CARmona, J. J. \& Necchi, O. (2002): Taxonomy and distribution of Paralemanea (Lemaneaceae, Rhodophyta) in Central Mexico. - Cryptogamie Algologie 23: 39-49.

Cyrus, B. (1947): Hydrobiologický průzkum vlivu přehrady u Seče na čistotu vody v řece Chrudimce. - Sb. St. hydrolog. TGM 1: 74-80.

Eloranta, P. \& Kwandrans, J. (1996): Distribution and ecology of freshwater red algae (Rhodophyta) in some Finnish rivers. - Nordic. J. Bot. 16: 107-117.

Eloranta, P. \& Kwandrans, J. (2002): Notes on some interesting freshwater Rhodophyta from Finland. Algological Studies 105: 95-109.

FILKIN, N. R. \& VIS, M. L. (2004): Phenology of Paralemanea annulata (Lemaneaceae, Rhodopyta) in an Ohio woodland stream. - Hydrobiologia 518: 159-168.

GardavskÝ, A., HindÁk, F. \& LhotskÝ, O. (1995): Sinice a riasy. - In: Kotlaba, F. (ed.): Červená kniha ohrozených a vzácných druhov rastlín a živočíchov SR a ČR 4: 7-29 pp., Príroda, Bratislava.

Hansgirg, A. (1886): Prodromus der Algenflora von Böhmen I. - Arch. Naturw. Landesdurchforsch. Böhmen, Prag 5(6): 1-288.

Hansgirg, A. (1892): Prodromus der Algenflora von Böhmen II. - Arch. Naturw. Landesdurchforsch. Böhmen, Prag 8(4): 1-262.

Israelson, G. (1942): The freshwater Florideae of Sweden. Studies on their taxonomy, ecology and distribution. - Symb. Bot. Upsal. 6(1): 1-134.

JohAnsson, C. (1982): Attached algal vegetation in running waters of Jämtland. Sweden. - Acta Phytogeogr. Suecica 71: 1-83.
KuČERA, P. \& MARVAN, P. (2004): Taxonomy and distribution of Lemanea and Paralemanea (Lemaneaceae, Rhodophyta) in the Czech Republic. - Preslia 76: 163-174.

Lederer, F. \& Lhotský, O. (2001): Přehled sladkovodních ruduch (Rhodophyta) v České republice. - In: Sbor. 17. sem. Aktuální otázky vodárenské biologie, 76-81 pp., VŠCHT, Praha.

LEDERER, F. \& SOUKUPOVÁ, L. (2002): Biodiversity and ecology of algae in mountain bogs (Bohemian Forest, Central Europe). - Algological Studies 144: 151-183.

LнотsкÝ, O. \& Rosa, K. (1955): Soupis moravskoslezských sinic a řas. -260 pp., ČSAV, Praha.

Maloch, F. (1937): Společenstva řas a rozsivek v jihozápadních Čechách. - Sborn. Prírodov. Klubu v Košiciach 3: 37-72.

Necchi, O. \& Zucchi, M. R. (1995): Record of Paralemanea (Lemaneaceae, Rhodophyta) in South America. Algological Studies 78: 33-38.

PAscher, A. (1903): Zur Algenflora des südlichen Böhmerwaldes. - Lotos 23: 161-211.

PoulíčKovÁ, A., LhotskÝ, O. \& DřímalovÁ, D. (2004): Prodromus sinic a řas České Republiky. - Czech Phycology 4: 19-33.

Sheath, R. G., Müller, K. M., Vis, M. L. \& Entwisle, T. J. (1996): A re-examination of the morphology, ultrastructure and classification of genera in the Lemaneaceae (Batrachospermales, Rhodophyta). Phycol. Res. 44: 233-246.

Starmach, K. (1977): Phaeophyta - Brunatnice, Rhodophyta - Krasnorosty. - In: Starmach, K. \& Sieminska, J. (eds): Flora słodkowodna Polski 14: 1-445 pp., Polska Akad. Nauk, Warszawa \& Krakow.

Vis, M. L. \& SheAth, R. G. (1992): Systematics of the freshwater red algal family Lemaneaceae in North America. - Phycologia 31: 164-179.

WÜNSCH, R. (1937): 7. Flerenliste. - In: GELING, R. \& WüNSCH, R. (eds): Die Pflanzendecke des Bezirkes Gablonz, 83-150 pp., Heimatkunde für den Landkreis Gablonz.

(C) Czech Phycological Society

Received August 23, 2007

Accepted January 12, 2008 